Baltic Astronomy, vol.22, 43-51, 2013

\title{
RADIO ASTRONOMICAL AND RADIOPHYSICAL STUDIES IN THE NEAR-EARTH SPACE IN THE RADIOPHYSICAL RESEARCH INSTITUTE
}

\author{
S. D. Snegirev, N. V. Bakhmetieva, N. A. Dugin, A. N. Karashtin and \\ V. P. Uryadov \\ Radiophysical Research Institute, B. Pecherskaya str., 25/12a, \\ Nizhny Novgorod,603950, Russia; sneg@nirfi.sci-nnov.ru
}

Received: 2012 November 13; accepted: 2012 November 28

\begin{abstract}
The paper describes possibilities of the experimental investigations of the near-Earth space by the facilities of the Radiophysical Research Institute at Nizhny Novgorod. We describe the experimental equipment, development of the research methods and some recent results.
\end{abstract}

Key words: techniques: radar astronomy, interferometric - instrumentation: detectors, interferometers - methods: observational - Earth: ionosphere - space vehicles

\section{INTRODUCTION}

The development of radioastronomical and radiophysical methods for the environmental studies of space is one of the most important problems being investigated in the Radiophysical Research Institute. The institute was a trailblazer of a method, which still remains among the most important methods in radio astronomy - the Very Long Baseline Interferometry, VLBI (Troitskii et al. 1975; Nechaeva et al. 2007).

In the 1980s the SURA facility for studying different ionospheric layers in the institute was created (Belov et al. 1983; Boiko et al. 1985). The SURA facility is intendent for different applied and fundamental researches of the Earth's upper atmosphere involving natural or artificial disturbances, simulation of radio wave propagation conditions in the SW and FM bands radio positioning of objects in the near space and broadcasting activities. The active experiments in the ionosphere are performed for detailed studies of dynamics of the ionospheric plasma, which is a propagation medium for decameter, meter and decimeter waves. The developed diagnostic methods make possible a detailed investigation of the ionospheric response to its disturbance by high-power radio-waves. Since the parameters of the ionospheric plasma vary with altitude and strongly depend on geophysical conditions, a variety of physical phenomena during the active experiments in the ionosphere take place, embracing a number of branches of plasma physics, astrophysics, geophysics, aeronomy and radio-wave propagation. All that 
allows to consider the ionosphere as a natural plasma laboratory, in which a wide range of applied and fundamental problems can be successfully solved (Boiko et al. 1985). It should be also emphasized that the experiments based on the action of the ionosphere by high-power radio beams give no pollution and environmental consequences.

The institute is also involved in the applied studies using the Russian linear frequency modulation network. The investigations are focused on the SW propagation and the communication stability in this range of radio waves (Ivanov et al. 2003).

The further content of the paper is divided into three sections describing our results in the listed research areas.

\section{VERY LONG BASELINE INTERFEROMETRY}

The Very Long Baseline Interferometry is an advanced method of radio astronomical and radio astrometric tasks was developed at RRI (earlier NIRFI) starting from the 1970s. The experts of the VLBI laboratory at RRI currently participate in a number of VLBI experiments in cooperation with the major Russian radio observatories and the radio telescopes in Ukraine, Latvia, Italy and China. The own VLBI system of RRI includes the receiver sites with antennas of different sizes at Staraya Pustyn, Zimenki and Nizhny Novgorod. The University of Nizhny Novgorod owns three complete receiving and transmitting systems, operating at frequencies of 1.6, 0.61 and $0.327 \mathrm{GHz}$.

The international collaboration in radio astronomy is aimed at the development and application of the VLBI methods for a wide range of scientific tasks (Nechaeva et al. 2007; Molotov et al. 2008; Gavrilenko et al. 2007; Dugin et al. 2011), such as:

- a radar investigation of the movement parameters of space debris objects and near-Earth asteroids;

- the study of physical parameters of the Solar wind plasma and the plasma of Earth's ionosphere in natural and artificially disturbed conditions radio raying it by the radio emission from the extragalactic radio sources and the signals of space satellites;

- the investigation of spatial structure of radio emission sources from the Solar flares.

During the last two years VLBI experiments on the reception of radio signals from the navigation satellites, such as GLONASS and GPS, were accomplished with different purposes: the distance diagnostics of the Earth ionosphere sounding it by satellite signals, the interferometer calibration and determination of parameters of the antenna and the receiving system at strong satellite signals, a precise determination of trajectories of the satellites.

\subsection{The VLBI radar method for the measurement of paths of the near-Earth asteroids and space debris}

The radar VLBI method was established theoretically in the Radiophysical Research Institute in the 1990s for a study of short-period variations in proper rotation of the Earth-group planets and the precise determination of their paths in the Radio Reference Frame coordinate system (Alekseev et al. 2000). Later on, the radar VLBI method has been applied for the determination of trajectory 
parameters of asteroids, crossing the Earth orbit (NEA) and for the space debris population in near-Earth space (Nechaeva et al. 2007; Molotov et al. 2008).

The method was experimentally validated in the international VLBI networks using the RT-70 planetary radar at Evpatoria (Ukraine). The method involves irradiating the objects under study with a signal from a high-power transmitter (monochromatic or sweep-frequency modulated) and receiving reflected signals by a set of antennas in the VLBI-mode, representing a combination of "classic" radio positioning of space objects (planets, asteroids and spacecrafts) and the VLBI reception of signals, reflected by these objects. Such a complex allows obtaining a unique tool: the radar allows to measure the range and range rate, while VLBI gives the angle and the angular velocity resolution. The main task of the experiments is the evaluation of object's coordinates and velocity on the measurements of time delay and Doppler frequency. Multiple VLBI receiving points, mutually spaced by thousands $\mathrm{km}$, permit to obtain a roughly equal linear resolution over all three spatial coordinates $(100-300 \mathrm{~m})$ for the inner planets, asteroids and artificial objects in the near-Earth space. For small objects of the Solar system, i.e. comets and asteroids, the used method permits a direct solution of the coordinates, since these objects have small angular dimensions and cannot be resolved by VLBIsystems. The accurate determination of the asteroid trajectory parameters allows to detect objects which are potentially dangerous to Earth.

Since 2001 a large number of observations of space objects by the radar VLBImethod was accomplished, including successful experiments on radar observations of Mars (2003), asteroid 2004XP14 (2006) and Venus (2007). An important aspect of this research project was the determination of exact paths of space debris. In 2001-2008 a series of accurate measurements of Doppler shift frequencies were obtained for tens of space debris objects. The results demonstrate that the radar VLBI experiments allow to reduce by several times the uncertainty of trajectories of the objects, preliminary measured by optical facilities. The proposed method for the VLBI reception of target signals from space debris at high elliptical orbits provides the precise Doppler shift measurements, which are used for improvement of orbital elements, sizes, rotation periods and inclinations of the rotational axis.

\subsection{VLBI research of turbulent plasma in the Earth's ionosphere by a method of radio raying}

Last year Radiophysical Research Institute and Ventspils International Radio Astronomy Centre organized investigations of the influence of ionospheric turbulence on the VLBI signals in decimetric wavelength range using the radio raying method. Compact extragalactic radio sources (at wavelength $90 \mathrm{~cm}$ ) and a transmitter of navigation space satellite (at wavelength $20 \mathrm{~cm}$ ) are used as the sources of radio emission for sounding of the medium. The main scientific task of this project is the determination of the ionospheric parameters, such as the spectral index of spatial spectrum and the velocity of irregularities of electron density.

The following VLBI stations took part in the first experiments: Irbene, RT-32 (Ventspils International Radio Astronomy Centre, Latvia), UNN, RT-2 (University of Nizhny Novgorod, Russia), RRI, Horn antenna and SURA, a complex for microwave diagnostics of the ionosphere (Radiophysical Research Institute, Nizhny Novgorod). As a result of the spectral correlation analysis, responses of the two-element interferometer RRI-UNN and the three-element interferometer RRI-UNN-Irbene were received for 12 satellites of the GLONASS and GPS nav- 
igation systems. Data processing for the determination of the turbulent medium parameters was implemented on a correlator NIRFI-4.

\section{THE SURA FACILITY FOR IRRADIATION OF THE IONOSPHERE}

The SURA facility is a unique multipurpose instrument for the near-Earth and space investigations, designed for a study of interaction of high-power radiation and the ionospheric and near-Earth plasma. It belongs to the worldwide network of application-dependent facilities, along with EISCAT (Tromso, Norway), HAARP (Alaska, U.S.A.), HYPAS (Alaska, U.S.A.) and SPEAR (Spitsbergen, the facility belongs to UK), being currently the only operating facility at mid-latitudes (the rest of them are located at polar latitudes). The instrumentation and equipment of the SURA facility is located in the Vasilsursk research base, about $140 \mathrm{~km}$ from Nizhny Novgorod and is operated by RRI.

The SURA facility operates in combination with many diagnostic devices, accommodated both in close vicinity to the transmitters and in the remote stations. The equipment at the SURA facility includes: BASIS, a computer-aided dynasonde (a new CADI dynasonde will be available soon), a partial reflection facility, a unit for the ionospheric research using the artificial periodic inhomogeneities, a receiver for observation of the artificial ionospheric radio emission, a mesosphere HF-radar, a unit for the radio and electro-acoustic atmospheric sounding, a set of radio astronomical receiving equipment, a set of equipment for the investigation of short electromagnetic pulses, auxiliary antennas and a feeder line. The stations at Zimenki remote diagnostic complex, $30 \mathrm{~km}$ form Nizhny Novgorod, and at Novaya Zhizn site, $30 \mathrm{~km}$ from Vasilsursk research base, contain the following equipment: a unit for the recording of signals from the vehicle-borne orbital satellite transmitters, a multifrequency complex for monitoring the solar processes, and an extremely low frequency and low-frequency receiving complex for measuring electromagnetic signals within the range of $0.01 \mathrm{~Hz}-100 \mathrm{kHz}$ ).

When compared with the foreign equivalents, the 144-element phased antenna array of SURA has one important advantage - it can be used both as a transmitting and a receiving antenna. This allows using the facility for research of the nearEarth plasma and the outer space objects by radio-sounding methods. The VDRT300 radio telescope created on the basis of SURA facility at present is a unique operating radio astronomical tool in the long-wave part of the decameter range. Details of the investigations carried out with the SURA facility can be found in the following review articles: Karashtin et al. (1999), Frolov et al. (2007) and Belikovich et al. (2007).

The development of radiophysical methods on SURA for the environmental studies during the last several years include: (1) the methods for diagnostics of the atmosphere and the ionosphere conditions in the presence of a high-power emission using the artificial periodic irregularities and (2) the method of resonant scattering of radio waves by artificial periodic irregularities (API).

Some active experiments in space, including those related to the exposure of the ionosphere to a high-power radio emission, inspired a development of new methods for ionosphere diagnostics, based on a controlled action on it. A new radiophysical method, developed at RRI, allows to make a significant progress in the investigations of the lower ionosphere, ionospheric D- and E-regions with the interlayer E-F valley, which could not be studied by the vertical sounding 
technique. The method is based on a generation of ionospheric plasma APIs in the field of a high-power standing wave, sounding them by probe radio waves (not affecting the propagation medium), reception of signals scattered by the periodic structure, recording their amplitude-phase characteristics, and measuring their altitude-temporal dependences.

The method for determination of a large number of the most important ionospheric parameters is based on the API formation theory developed at RRI. The application of the method permits us to obtain information about the following ionosphere and neutral atmosphere characteristics:

- electron density profiles from the lower part of the D-region up to the altitude of the F-region maximum;

- vertical motion velocities at the altitudes of the D- and E-regions;

- atmospheric temperature and density at the altitudes of the E-region;

- the ion composition and the concentration of basic ions in the sporadic Elayer;

- fractional concentration of negatively charged oxygen ions in the D-region;

- electron and ion temperatures in the F-region;

- the following turbulence parameters: vertical component of the turbulent velocity, the turbulent diffusion coefficient and the turbopause altitude;

- irregular structure of the lower ionosphere, including the stratification of the regular E-region, sporadic ionization layers, additional layers at the electron density profiles;

- parameters of internal gravity waves and their spectral characteristics.

In recent years, the method of the resonant scattering has been improved. A new method of the electron density measurement in the E-region by generation of the periodic irregularities with two special scales (two-frequency method) has been theoretically validated. In the first experiments the profiles of electron density in the range of altitudes $90-120 \mathrm{~km}$ were received (Belikovich et al. 1986). The API were created by the power emission from the SURA heating facility.

The viscous and thermal conductivity effects on the propagation of the internal gravity waves have been examined determining characteristics of the neutral atmosphere at the E-region altitudes using the API technique. Internal gravity waves with periods from 5-10 minutes to 4 hours have been observed when measuring the temperature and density variations in neutral atmosphere, as well as the vertical plasma velocity, which coincides with the neutral component velocity at the mentioned altitudes. Numerical estimates of the internal gravity wave dissipation at the altitudes $\sim 100 \mathrm{~km}$ due to the above factors have been made. More results of the atmospheric parameter measurements by this method are given by Belikovich et al. (2002) and Bakhmet'eva et al. (1998, 2010).

Except of the investigation of the action on the ionospheric plasma by a powerful HF radiation, the SURA facility is used for studies of the ionospheric D-region (altitudes from 50 to $90 \mathrm{~km}$ ) using the partial reflection technique. This method is based on radio location of the lower ionosphere and receiving signals from two magnetoionic components, scattered by the natural plasma irregularities.

In the late 1960s, the partial reflection technique was introduced in NIRFI. The method was intensively used in the 1980s. Over 2000 profiles of electron density in different helio-geophysical conditions have been obtained. An empirical model of the altitude profile of the electron density at the midlatitude ionospheric D- 
region has been developed. Altitude profiles of the electron density and electron loss in the periods of solar flares were obtained. The advanced computerized technologies for the signal processing allowed us to use the coherent accumulation method, reducing the noise level by $15-20 \mathrm{~dB}$. The theory of the partial reflection technique has been developed in detail.

In recent years, the new data in studies of the lower ionosphere were obtained. It was found that electron density in the D-region in quiescent and disturbed conditions is proportional to the intensity of the ionizing radiation, $N \sim q$, rather than $N \sim q^{0.5}$, as given by dominating aeronomical theories. We have proposed a hypothesis which suggests that the basic electron loss process in the D-region should be due to recombination of electrons and ions on aerosol and dust particles with a size of $l \approx 10^{-5} \mathrm{~cm}$ and with a dust concentration of $N \approx 30-100 \mathrm{~cm}^{-3}$, rather than with the dissociative recombination. Distinctive features of polar and mid-latitude ionosphere are studied in cooperation with the Polar Geophysical Institute, Kola Science Center, Russian Academy of Sciences, which has a partial reflection facility with the similar parameters. In recent years, the partial reflection technique was further developed by applying it in combination with the method of scattering of radio waves by artificial periodic irregularities of the ionospheric plasma. The experience of RRI has been used in development of the partial reflection facilities in other research institutes. The partial reflection technique can be successfully used for the lower ionosphere monitoring. Important aspects of these methods are described by Belikovich et al. (1986, 2007).

The simultaneous studies of the dynamics of artificial decameter inhomogeneities (within a range of the transverse geomagnetic field $l_{\perp} \sim 7-125 \mathrm{~m}$ ) and the artificial diagnostic radio emission of the ionosphere in the heating experiments with the SURA facility were performed at a number of remote sites of reception of the scattered signals. The diurnal behavior of the development and relaxation of the inhomogeneities was analyzed by comparing them with the diagnostic artificial ionospheric radio emission. Comparing the day and night measurements of inhomogeneities, we have detected a simultaneous increase of their relaxation time by a factor of 5 to $10 \mathrm{~s}$ (up to $100 \mathrm{~s}$ ), and the diagnostic radio emission. This effect can be related to the growth of the lengths of the inhomogeneities by factor of 2 to 3 during their relaxation in the longitudinal diffusion mode.

A new magnetosphere sounding method for control of the magntospheric LF wave propagation has been proposed and verified experimentally. It involves the application of high-power SW radiation to create disturbances in the ionospheric F-region, which are responsible for a rise of increased density regions, so called ducts.

A method for the determination of characteristics of the artificial ionospheric turbulence has been developed. The turbulence is generated by a powerful SW emission at the altitudes of the outer ionosphere $(h \geq 600 \mathrm{~km})$. The method uses a diagnostic equipment, installed on earth satellites.

\section{A NETWORK FOR THE LINEAR FREQUENCY MODULATION IN OBLIQUE SOUNDING OF THE IONOSPHERE}

The short-wave communication plays an important role in the research of the ionospheric wave propagation. For the control a radio line frequency resource (in the case when the communication channel is unstable), a permanent monitoring 
of current ionospheric conditions and a channel noise level control are necessary. The ionospheric channel diagnostics allows to estimate optimal frequency-time intervals, fading (multipath propagation) to provide a high communications reliability. In the last two decades low-power oblique-incidence chirp sounders (tens to hundreds of watts) have been used.

The study of propagation of radio waves in the natural or artificially disturbed ionosphere using a special ionosonde and direction finder with a linear frequency modulation has been done in the following sequence.

- The multi-window spectral analysis method was chosen.

- The day-and-night measurements of the parameters of ionospheric radio channel with the oblique-incidence linear frequency modulation, sounding paths of different length and orientation, were performed.

- The results of two experiments on short wave propagation along the transequatorial, sub-auroral and midlatitude paths, made using a linear frequency modulation ionosonde direction finder were analyzed, abnormal signals found, and their propagation mechanisms determined;

- An approach for the determination of parameters of the F-region in the horizontal inhomogeneous ionospheric model using the oblique incidence sounding results has been developed;

- The results of measuring structure and dynamics of small-scale artificial ionospheric turbulence, in particular, new results on the diagnostics of artificial ionospheric turbulence have been obtained. A cluster structure of artificial ionospheric turbulence has been discovered.

The methods of on-line forecasting the ionospheric conditions using a linear frequency modulation sounding to provide stable and reliable short-wave communications received further development.

To provide efficient operation of different purpose radioelectronic systems an up-to-date hardware and software complex for online determination of optimal operating frequencies of the ionospheric radio-channel based on the linear frequency modulation ionosonde has been developed in RRI in cooperation with the Southern Federal University (Vertogradov et al. 2010a,b).

Unlike traditional devices, the developed complex permits determining such important parameter, as the ionospheric coefficient of haze (reflected / scattered signal ratio). This considerably extends the capabilities for the determination of key characteristics of the ionospheric channel for different channel types (Rician, Rayleigh etc.).

Using the data obtained by the hardware and software complex the following characteristics can be obtained:

- the range-frequency and amplitude-frequency response of the ionospheric channel;

- the lowest and maximum observed frequencies;

- the number of paths;

- the intervals of multipath propagation;

- the signal-to-interference ratio at all signal transmission frequencies for all propagation modes;

- the ionospheric coefficient of haze;

- the bit error probability for different types of communication signals;

- the communication reliability. 
Therefore, the developed hardware and software complex represents a solution for online determination of operating frequencies for a radio communication line and can be used in the ionospheric wave and frequency allocation service for a dynamic control of the frequency resource of radio lines in the decameter range.

\section{CONCLUSIONS}

An extensive base for experimental studies of the ionosphere has been established in the Radiophysical Research Institute, including the multipurpose SURA facility for investigations of the near-Earth space. It is one of the main instruments of the Russian network of Linear Frequency Modulation for the oblique sounding of the ionosphere. The experimental studies in the institute apply the modern methods such as the Very Long Baseline Interferometry (VLBI), based on the international network of radio telescopes and the methods of active modification of the ionosphere by SW radiation. The main results of the research can be summarized in the following items.

(1) The VLBI radar method was experimentally validated in an international network using the RT-70 planetary radar in Evpatoria (Ukraine). Since 2001 a large number of observations of space objects by the radar VLBI method were organized, including the successful radar observations of Mars (2003), the asteroid $2004 X P 14$ (2006) and Venus (2007).

(2) The methods for diagnostics of the atmosphere and the ionosphere conditions in the presence of a high-power emission using artificial periodic irregularities have received further development using the SURA facility.

(3) The partial reflection technique for the determination of electron density profiles within the altitude interval from 70 to $90 \mathrm{~km}$ has been updated.

(4) A new method of for magnetosphere sounding to control the magntospheric LF wave propagation has been proposed and verified experimentally.

(5) The methods of on-line forecasting of ionospheric conditions using the linear frequency modulation sounding to provide stable and reliable short-wave communications received further development.

ACKNOWLEDGMENTS. The investigations were partly supported by Russian Foundation of Basic Researches (projects 99-05-64464, 00-05-64695, 01-0564504, 02-05-65281, 04-05-64140, 05-05-64304, 05-05-64111, 08-02-97036, 09-0500450, 11-02-97028, 12-02-00177). We also acknowledge the input by the project 97-05-64392 common with the Deutsche Forschungsgemeinschaft Researches. The research was also partly supported by the State contract P683 (01201061443, 10.09.2010).

\section{REFERENCES}

Alekseev V. A., Lipatov B. N., Reznikova V. A. 2000, Radiophysics and Quantum Electronics, 43, 607

Bakhmet'eva N. V., Belikovich V. V., Benediktov E. A. et al. 1998, Radio Science, 33,583

Bakhmet'eva N. V., Belikovich V. V., Egerev E. N., Tolmacheva A. V. 2010. Radiophysics and Quantum Electronics, 53, 63

Belikovich V. V., Grach S. M., Kerashtin A. N., Kotik D. S., Tokarev Yu. V. 2007, Radiophysics and Quantum Electronics, 50, 497 
Belikovich V. V., Benediktov E. A., Goncharov N. P., Grishkevich L. V., Vyakirev. V. D. 1986, J. Atmos. Terr. Phys., 48, 1241

Belikovich V. V., Benediktov E. A., Tolmacheva A. V., Bakhmet'eva N. V. 2002, Ionospheric Research by Means of Artificial Periodic Irregularities, Copernicus GmbH, Katlenburg-Lindau, Germany, 160 pp.

Belikovich V. V., Vyakhirev V. D., Kalinina E. E. 2007, Geomagnetism and Aeronomy, 47, 72

Belov I. F., Bichkov V. V., Getmantsev G. G., Mityakov N. A., Pashkova G. R. 1983, The Experimental Facility SURA for Investigation of Artificial Disturbances of Ionosphere, Gorkii, NIRFI, Preprint No. 167, 28 p.

Boiko G. N., Erukhimov L. M., Zyuzin V. A. et al. 1985, Radiophysics and Quantum Electronics, 28, 259

Dugin N. A., Nechaeva M. B., Antipenko A. A., Dement'ev A. F., Tikhomirov Yu. V. 2011, Radiophysics and Quantum Electronics, 54, 159

Frolov V. L., Bakhmet'eva N. V., Belikovich V. V. et al. 2007, Uspekhi Fizicheskikh Nauk, 177, 330

Gavrilenko V. G., Nechaeva M. B., Pushkarev A. B. et al. 2007, Radiophysics and Quantum Electronics, 50, 253

Ivanov V. A., Kurkin V. I., Nosov V. E. et al. 2003, Radiophysics and Quantum Electronics, 46, 821

Karashtin A. N., Komrakov G. P., Tokarev Yu. V., Shlyugaev Yu. V. 1999, Radiophysics and Quantum Electronics, 42, 674

Molotov I., Nechaeva M., Falkovich I. et al. 2008, in Measuring the Future, Proc. of Fifth IVS General Meeting, eds. A. Finkelstein \& D. Behrend, Nauka Publishers, p. 30

Nechaeva M. B., Antipenko A. A., Dement'ev A. F. et al. 2007, Radiophysics and Quantum Electronics, 50, 527

Troitskii V. S., Alekseev V. A., Nikonov V. N. 1975. Sov. Phys. Uspekhi, 18, 832

Vertogradov G. G., Uryadov V. P., Vertogradova E. G. 2010. The Device for Definition of Optimum Working Frequencies of Ionospheric Radio Channel, Patent No. 2394371 of Russian Federation

Vertogradov V. P., Uryadov V. P., Vertogradov V. G. et al. 2010, Journal of Radio Electronics, No. 12 (http://jre.cplire.ru) 